\section{$\underset{\text { hommes }}{\text { \& migrations }}$}

\section{Hommes \& migrations}

Revue française de référence sur les dynamiques

migratoires

$1281 \mid 2009$

France-Brésil sous l'angle des migrations et de l'altérité

\title{
Miroir et mémoire du Brésil
}

Le roman de Julie Delafaye-Bréhier

\section{Andréa Borges Leão}

Traducteur : Julien Zeppetella

\section{(2) OpenEdition}

\section{Journals}

\section{Édition électronique}

URL : http://journals.openedition.org/hommesmigrations/405

DOI : 10.4000/hommesmigrations.405

ISSN : 2262-3353

Éditeur

Musée national de l'histoire de l'immigration

\section{Édition imprimée}

Date de publication : 1 septembre 2009

Pagination : 180-184

ISSN : 1142-852X

\section{Référence électronique}

Andréa Borges Leão, « Miroir et mémoire du Brésil », Hommes \& migrations [En ligne], 1281 | 2009, mis en ligne le 29 mai 2013, consulté le 22 septembre 2020. URL : http://journals.openedition.org/ hommesmigrations/405; DOI : https://doi.org/10.4000/hommesmigrations.405

Ce document a été généré automatiquement le 22 septembre 2020.

Tous droits réservés 


\title{
Miroir et mémoire du Brésil
}

\author{
Le roman de Julie Delafaye-Bréhier
}

\author{
Andréa Borges Leão
}

Traduction : Julien Zeppetella

1 À l'époque de l'Empire du Brésil, les livres de voyage étaient les préférés des lecteurs brésiliens. Le français Baptiste-Louis Garnier, qui migra à Rio de Janeiro en 1844 et se spécialisa dans le commerce du livre importé, avait dans un de ses catalogues de l'année 1858 presque deux cents titres classés sous les rubriques "souvenirs", "chroniques", "anecdotes", "géographies", "voyages" et "descriptions". Dans beaucoup de ces ouvrages, le Brésil apparaissait comme l'un des thèmes privilégiés.

2 La littérature de voyage attirait la curiosité pour le pittoresque de l'aventure, mettant en exergue le courage des voyageurs - découvreurs, explorateurs ou simples touristes - face aux intempéries de la traversée, et proposait des descriptions romancées des modes de vie et des croyances de peuples inconnus (presque toujours des Indiens des Amériques). $\mathrm{Au}$ XIXe siècle, ce type de littérature rejoint les textes destinés au public juvénile. Dans des pays comme la France, les bibliothèques d'éducation morale et de formation religieuse commencent à inclure des titres qui se distinguent par leur traitement des peuples "sauvages", offrant ainsi une forme d'instruction qui ne représente pas de danger pour la foi chrétienne des jeunes lecteurs français. En effet, à la fin de l'histoire, les Indiens "sauvages" sont convertis au catholicisme, au travers du baptême ou du mariage chrétien.

\section{Le Brésil dans les livres de jeunesse français : des vertus pédagogiques}

3 Les descriptions méthodiques des cinq continents, les cartes, globes et traités de géographie stimulaient l'intérêt pour les sciences de la nature et conviaient les lecteurs à visiter les musées et cabinets de curiosités. Par ailleurs, les serpents, monstres et festins anthropophages devaient aiguiser les peurs et satisfaire la curiosité des enfants, mais 
aussi stimuler l'apprentissage des différences. En terme de projet éditorial, les bibliothèques de morale et d'éducation religieuse furent affectées principalement à la vie des Indiens du Brésil. Les coutumes des hommes américains motivaient des réflexions sur les excès provoqués par la barbarie - tels que la pratique de l'anthropophagie. Elles pouvaient même les exclure des domaines de la "civilisation" aux yeux des lecteurs européens, lesquels, en même temps, rangeaient ces ouvrages parmi leurs lectures préférées. Ces thèmes figuraient dans les collections des livres de jeunesse de la librairie parisienne des frères Garnier. Et, une fois que le goût du public français pour la littérature de voyage fut avéré, ces titres ont été exportés vers le Brésil.

Pour les lecteurs européens, ils représentaient une porte sur la différence. Mais, pour les lecteurs brésiliens, ils pouvaient fonctionner comme un miroir de la mémoire. Un univers culturel commun reliait les communautés de jeunes lecteurs de l'Ancien et du Nouveau Monde. C'est dans ce contexte que des lecteurs d'une culture en rencontrent une autre'.

\section{Le livre de voyage : un roman de formation morale}

5 Le genre catalogué "voyage" finit par faire partie du roman de formation morale. Pour les auteurs de fiction juvénile, les descriptions sont appropriées et interviennent comme références et contre-références de modèles moraux dans les étapes de l'éducation des jeunes lecteurs français. Les objectifs du roman moral sont explicites: l'application des principes chrétiens au travers des actions modèles des personnages. Le roman moral se définit comme une littérature spirituelle, divertissante et instructive. Ces ouvrages visent à produire une sensibilité engagée dans la croyance et ils sont soumis à l'aval de comités ecclésiastiques de lecture avant publication. Ces derniers, avec leur fonction de premiers censeurs, inaugurent un système juridico-religieux de contrôle des textes. Les éditeurs Mégard de la ville de Rouen (grands distributeurs de livres de collections enfantines dans toute la France et, en particulier, via les frères Garnier, diffuseurs de la littérature française au Brésil) ne se dispensaient pas de cet examen préliminaire des autorités responsables de l'éducation religieuse. En prenant en considération les objectifs de l'Église catholique - une caractéristique du régime de production éditoriale de cette époque -, cette pratique indique une soumission à ce que Jean-Yves Mollier nomme la "logique de la demande sociale".

\section{Le poids des femmes de lettres dans la littérature de formation}

6 Afin de mettre la moralité en action, il était nécessaire d'avoir l'adhésion des femmes de lettres. Un regard austère, posé sur le Brésil et son système de relations coloniales par une partie des femmes de lettres, illustre bien le poids de la position féminine dans le nouveau régime de production du roman moral. Connaissant de nombreuses rééditions depuis 1847 par les libraires Mégard et Mame dans les collections Bibliothèque morale de la jeunesse et Bibliothèque de la jeunesse chrétienne, le livre de l'auteure allemande Amélie Schoppe Les émigrants au Brésil en constitue un bon exemple.

7 Le roman Les Portugais d'Amérique : souvenirs historiques de la guerre du Brésil en 1635 (contenant un tableau intéressant des mœurs et usages des tribus sauvages, des détails instructifs sur la situation des colons dans cette partie du Nouveau Monde) de Julie 
Nicolase Delafaye-Bréhier est celui qui associe le mieux un point de vue sur le système colonial aux ingrédients de la littérature de formation.

8 L'analyse de ce roman, inspirée de la méthodologie et des théories de l'histoire culturelle ${ }^{3}$ , et l'observation de son contexte de diffusion, devraient ainsi éclairer la façon dont se construisent les significations des œuvres et les processus de diffusion de l'édition française au Brésil.

9 Ce livre obtient l'approbation de l'archevêché de Paris le 23 octobre 1846. Deux mois plus tard, il est inscrit à ce que l'on appelle la Bibliographie de la France ou Journal général de l'imprimerie et de la Librairie ${ }^{4}$, puis est définitivement publié par la maison Lehuby en 1847. Il est classé dans les œuvres à destination de la jeunesse et connaît deux rééditions successives durant sa première année de publication.

Le succès des Portugais en Amérique atteste de la popularité du genre dans lequel il a été classé. En 1858, soit un peu plus de dix ans après sa première édition parisienne, il entre dans la bibliothèque des œuvres instructives et récréatives de la librairie Baptiste-Louis Garnier, la filiale à Rio de Janeiro de la maison-mère française.

\section{Le Brésil de Julie Delafaye-Bréhier : la vengeance des colonisés}

11 Julie Nicolase Delafaye-Bréhier est née en 1780 à Nantes. Éduquée dans la plus stricte tradition catholique, à treize ans, au milieu des querelles religieuses qui agitent la France révolutionnaire, elle se convertit au protestantisme. À trente deux ans, elle commence à publier une œuvre littéraire vaste et populaire destinée aux enfants et aux jeunes. C'est à cette période, où le marché du livre de jeunesse est dominé par les éditions catholiques, que la catégorie "Femme de lettres" s'affirme. Son parcours littéraire n'ouvre pas seulement une discussion autour du droit d'auteur en ce qui concerne les femmes de lettres, mais aussi de nouvelles pistes sur les réseaux de production, dans la France du XIX ${ }^{e}$ siècle, d'œuvres de fiction sur le Brésil.

12 Dans Les Portugais en Amérique, l'auteure situe son histoire dans la ville d'olinda (dans l'État du Pernambouco au Brésil) en 1635, période de l'occupation hollandaise dans le Nordeste du Brésil. Les personnages sont des colons portugais, des Indiens Tapuyas et des Noirs insoumis de la République de Palmares. Le texte narre l'exécution d'un plan de vengeance - enlèvement suivi d'une captivité dans la forêt tropicale - élaboré par les Indiens contre leurs maitres et bourreaux, les colons portugais. Deux dames, Elvire et Héléna, sont enlevées par leurs esclaves domestiques, la vieille Mocap, instigatrice du plan, et la jeune métisse Yassi-Miri, nourrice du petit Sébastien, fils d'Elvire. Arraïp, l'esclave attaché personnellement à Don Aleixo, le mari d'Elvire, participe aussi au complot.

13 Profitant de la confusion causée par l'arrivée des Hollandais lors de l'occupation de la ville, Mocap fuit avec les deux femmes, ainsi qu'avec Yassa-Miri et Sébastien, en direction de la tribu des Tapuyas. Seule la vieille Tupinamba connaît les méandres de la forêt, de sorte que son retour avec les deux maitresses captives sera la preuve de son triomphe et de sa conquête.

14 Pendant ce temps, Don Aleixo, accompagné d'Arraïp, se dirige vers le fort Matias d'Albuquerque. Après de longs débats théologiques avec son esclave - ce dernier lui 
explique que toutes les créatures sont les enfants du même Dieu, ce qui est contradiction avec le fait de capturer des Indiens et de leur infliger de mauvais traitements -, le noble portugais est fait prisonnier par des Noirs insoumis de la République de Palmares. Il est le témoin de l'organisation d'une république tropicale avec des droits et des devoirs, mais s'horrifie devant les libations et les excès des fêtes lors de la récolte du maïs.

Don Aleixo arrive à fuir, mais rencontre un groupe d'Indiens féroces qui le capture. Cette fois, le noble portugais est fait prisonnier en vue de servir de met lors d'un festin cannibale. Sur le point d'être dévoré, il va même jusqu'à lancer des pierres sur ses exécuteurs - selon les coutumes narrées par les voyageurs du XVI ${ }^{\mathrm{e}}$ siècle ${ }^{5}-$, avant d'être sauvé par un missionnaire jésuite. Il rencontre à nouveau Arraïp et découvre la trahison.

Durant une longue journée dans la forêt tropicale, affrontant serpents, monstres et cours d'eau, les deux dames vont confronter leurs valeurs à celles des Tapuyas, s'appuyant sur les préceptes de la religion catholique, la foi dans les sacrements et l'inexorable conversion des barbares américains. Les deux esclaves fugitives, habitées par un fort sentiment de vengeance, que la narratrice explique en termes d'"obligation envers la dignité" perdue lors des mauvais traitements reçus en esclavage, se réjouissent de la nouvelle situation.

À Olinda, Héléna menait la vie lascive des colons portugais. Née au Brésil, fille d'un maître de plantation ruiné, elle est très dure avec les esclaves. Tandis qu'Elvire, née au Portugal, est le modèle de la bonne chrétienne. Elle va même jusqu'à accorder son pardon aux sauvages qui la séquestrent.

\section{Le récit de fiction : un lieu de reversement du rapport colon/colonisé}

18 Durant leur captivité, les deux dames portugaises réduites en esclavage sont confrontées à deux graves problèmes d'ordre moral et religieux. Le premier est en rapport avec l'éducation du petit Sébastien qui devrait, selon les nouvelles coutumes, se faire percer la lèvre inférieure et l'orner d'une pierre bleue. Pour sa mère, cet acte s'apparente à une mutilation. Le chef des Tapuyas, sorte de véritable sultan sauvage, s'éprend de la portugaise Héléna et désire en faire sa septième épouse. Cependant, comment une chrétienne pourrait-elle se marier avec un homme déjà marié six fois ? Profitant du fait qu'Héléna se soit éloignée du camp pour aller chercher de l'eau au fleuve, les autres épouses du chef, inquiètes de l'imminence de leur perte de position au profit d'une étrangère, l'enlèvent, la torturent et la trainent par les cheveux avant de l'attacher à un tronc d'arbre près d'une rivière fréquentée par des serpents venimeux. Après la disparition d'Héléna, le chef, plein de colère, expulse de la tribu Mocap et sa dernière captive, Elvire.

Les personnages passent à nouveau par un chemin semé d'embûches, mais cette fois à travers le désert en direction de la ville d'Olinda. Mocap meurt de soif durant la traversée, non sans s'être fait auparavant baptiser par Elvire. Cette dernière, accompagnée de YassiMiri et de Sébastien, est retrouvée par Don Aleixo. Des années plus tard, Héléna aussi sera retrouvée, démente et vivant dans le désert au sein d'une famille hollandaise. 


\section{Conclusion}

20 L'histoire des Portugais en Amérique conduit les lecteurs à questionner des rôles sociaux qui ne sont pas figés indéfiniment. L'histoire coloniale peut aussi être écrite à l'envers. Les règles de dépendance et d'assimilation des colonisées par rapport aux colonisateurs peuvent être déplacées. La narration de la captivité des maîtres portugais chez les Tapuyas finit par révéler un système de contradictions qui culmine avec l'effondrement du monde de certitudes de la colonie portugaise au Brésil, bien que cet effondrement soit limité du fait du triomphe final du christianisme.

21 Le plus remarquable dans cette histoire est qu'elle ait été écrite quelques années avant que l'écrivain brésilien José de Alencar ne publie le roman 0 guarani (1857), considéré comme pionner dans l'invention de l'indianisme brésilien.

\section{NOTES}

1. M. de Certeau, L'écriture de l'histoire, 1975.

2. J.-Y. Mollier, "Librairie et imprimerie à l'époque de la révolution industrielle", in Revue française d'histoire du livre, $\mathrm{n}^{\circ} 100$ - 101, Société des bibliophiles de Guyenne, 1998.

3. Roger Chartier, História Cultural, entre práticas e representações, Rio de Janeiro ; Editora Difel, 1990.

4. Bibliographie de la France était un livre dans lequel les éditeurs français déclaraient toutes leurs publications.

5. En divers passages, nous rencontrons les célèbres descriptions de Jean de Léry dans son livre Histoire d'un voyage faict en la terre du Brésil (1 ${ }^{\text {ère }}$ éd. 1578) et d'André Thevet dans Les singularités de la France antarctique, autrement nommée Amérique, et de plusieurs terres et isles découvertes de nostre temps (1 $1^{\text {ère }}$ éd. 1557).

\section{RÉSUMÉS}

Au XIX $\mathrm{X}^{\mathrm{e}}$ siècle, le livre est au cœur du processus d'échange culturel entre la France et le Brésil. Le roman d'aventure brésilien irrigue alors le marché éditorial français. Repris par les romanciers français, le genre est mis au service de l'instruction de la jeunesse hexagonale. En retour, les romans français inspirés par la littérature brésilienne sont diffusés au Brésil. Voici comment le va-et-vient des représentations tend à ce pays d'Amérique du Sud un miroir de sa propre histoire. 
AUTEURS

ANDRÉA BORGES LEÃO

UFC, Brésil 